\title{
Criminologie
}

\section{Analyse du processus d'empowerment dans des trajectoires de femmes victimes de violence conjugale à travers le système judiciaire}

\author{
Dominique Damant, Jo Bélanger et Judith Paquet
}

Volume 33, numéro 1, printemps 2000

La victimologie : quelques enjeux

URI : https://id.erudit.org/iderudit/004716ar

DOI : https://doi.org/10.7202/004716ar

Aller au sommaire du numéro

Éditeur(s)

Les Presses de l'Université de Montréal

ISSN

0316-0041 (imprimé)

1492-1367 (numérique)

Découvrir la revue

Citer cet article

Damant, D., Bélanger, J. \& Paquet, J. (2000). Analyse du processus d'empowerment dans des trajectoires de femmes victimes de violence conjugale à travers le système judiciaire. Criminologie, 33(1), 73-95. https://doi.org/10.7202/004716ar
Résumé de l'article

Bien qu'un nombre important de recherches, pourtant diversifiées, aient été réalisées auprès de femmes victimes de violence conjugale, les études portant spécifiquement sur les femmes qui ont recours au système judiciaire se font plutôt rares. Les écrits consultés ont permis d'identifier entre autres des facteurs facilitant le recours au processus judiciaire et des facteurs le freinant. Plus récemment, d'autres études ont porté de façon plus particulière sur les liens pouvant exister entre le fait pour une victime de s'engager dans le système judiciaire et son processus d'empowerment. L'étude qui est l'objet du présent article s'est intéressée de façon particulière au processus d'empowerment de femmes victimes de violence conjugale qui ont eu recours au système judiciaire. Elle porte sur l'analyse de 29 entrevues semi-dirigées avec des femmes victimes de violence conjugale, engagées ou non dans un processus judiciaire. Le paradigme que nous avons retenu pour l'analyse des données est le paradigme structurel. Trois caractéristiques généralement liées à la définition de la violence faite aux femmes ont déterminé ce choix : la violence est située dans un contexte de relations de pouvoir caractérisées par la domination ; le caractère discriminant de ces relations de pouvoir est lié au fait d'appartenir à un sexe plutôt qu'à un autre ; la dimension sociale et publique du problème de la violence est reconnue.

Le modèle de l'empowerment tel que nous l'avions élaboré semble approprié pour l'étude de trajectoires de femmes victimes de violence conjugale et laisse présager que toute démarche d'aide peut aider les femmes à s'engager dans un processus d'empowerment. Nos données nous permettent également d'identifier des éléments qui facilitent ce processus : support émotionnel ou informationnel. Nous n'avons toutefois pas pu cerner des éléments spécifiques au système judiciaire, en tant qu'institution sociale, qui favoriseraient le processus d'empowerment. Toutes les répondantes ont identifié des facteurs aidants et des obstacles. Seul le discours de nature plus sociale des répondantes différencie celles qui ont complété le parcours dans le système judiciaire des autres répondantes. L'hypothèse que nous retenons à ce moment-ci est que le fait de mener à terme des démarches judiciaires est plutôt indicateur d'empowerment. Si ceci s'avérait juste, on devrait en conclure que quelles que soient les décisions prises par les femmes à toutes les étapes du processus judiciaire, celles-ci doivent être respectées. Par ailleurs, l'information donnée, tout particulièrement en maison d'hébergement, qui analyse la violence conjugale comme un problème social et qui cherche à développer un mouvement de solidarité entre les femmes, semble être un facteur important dans le processus d'empowerment identifié dans cette étude.

Nous croyons que l'utilisation du modèle du processus d'empowerment que nous avons élaboré peut être un apport intéressant en ce qui concerne l'intervention auprès des femmes victimes de violence conjugale. Le modèle permet d'identifier l'étape du processus à laquelle la victime se situe et les besoins qu'elle manifeste (émotifs, cognitifs, comportementaux). On pourra alors lui proposer un type d'aide et d'informations plus pertinent à ses besoins. L'utilisation de ce modèle offre aussi l'avantage de comprendre qu'il n'est peut-être pas le moment de proposer à une femme d'entreprendre une démarche légale et qu'elle n'est peut être pas prête à persévérer en ce sens.
Tous droits réservés (C) Les Presses de l'Université de Montréal, 2000
Ce document est protégé par la loi sur le droit d'auteur. L’utilisation des services d’Érudit (y compris la reproduction) est assujettie à sa politique d'utilisation que vous pouvez consulter en ligne.

https://apropos.erudit.org/fr/usagers/politique-dutilisation/ 


\section{Analyse du processus d'empowerment dans des trajectoires de femmes victimes de violence conjugale à travers le système judiciaire}

Dominique Damant

professeure et chercheure

École de service social

Université Laval • Canada

Équipe VICTOIRE ${ }^{1}$ du CRI-VIFF',

dominique.damant@svs.ulaval.ca

Jo Bélanger

professionnelle de recherche

CRI-VIFF

jo.belanger@fss.ulaval.ca
Judith Paquet

professionnelle de recherche CRI-VIFF

judith.paquet@fss.ulaval.ca

RÉSUMÉ - Bien qu'un nombre important de recherches, pourtant diversifiées, aient été réalisées auprès de femmes victimes de violence conjugale, les études portant spécifiquement sur les femmes qui ont recours au système judiciaire se font plutôt rares. Les écrits consultés ont permis d'identifier entre autres des facteurs facilitant le recours au processus judiciaire et des facteurs le freinant. Plus récemment, d'autres études ont porté de façon plus particulière sur les liens pouvant exister entre le fait pour une victime de s'engager dans le système judiciaire et son processus d'empowerment. L'étude qui est l'objet du présent article s'est intéressée de façon particulière au processus d'empowerment de femmes victimes de violence conjugale qui ont eu recours au

1. VICTOIRE : Violence conjugale : transformer et orienter par l'intervention et la recherche. Équipe subventionnée par le Conseil québécois de la recherche sociale (CQRS).

2. Centre de recherche interdisciplinaire sur la violence familiale et la violence faite aux femmes. 
système judiciaire. Elle porte sur l'analyse de 29 entrevues semi-dirigées avec des femmes victimes de violence conjugale, engagées ou non dans un processus judiciaire. Le paradigme que nous avons retenu pour l'analyse des données est le paradigme structurel. Trois caractéristiques généralement liées à la définition de la violence faite aux femmes ont déterminé ce choix : la violence est située dans un contexte de relations de pouvoir caractérisées par la domination ; le caractère discriminant de ces relations de pouvoir est lié au fait d'appartenir à un sexe plutôt qu'à un autre ; la dimension sociale et publique du problème de la violence est reconnue.

Le modèle de l'empowerment tel que nous l'avions élaboré semble approprié pour l'étude de trajectoires de femmes victimes de violence conjugale et laisse présager que toute démarche d'aide peut aider les femmes à s'engager dans un processus d'empowerment. Nos données nous permettent également d'identifier des éléments qui facilitent ce processus : support émotionnel ou informationnel. Nous n'avons toutefois pas pu cerner des éléments spécifiques au système judiciaire, en tant qu'institution sociale, qui favoriseraient le processus d'empowerment. Toutes les répondantes ont identifié des facteurs aidants et des obstacles. Seul le discours de nature plus sociale des répondantes différencie celles qui ont complété le parcours dans le système judiciaire des autres répondantes. L'hypothèse que nous retenons à ce moment-ci est que le fait de mener à terme des démarches judiciaires est plutôt indicateur d'empowerment. Si ceci s'avérait juste, on devrait en conclure que quelles que soient les décisions prises par les femmes à toutes les étapes du processus judiciaire, celles-ci doivent être respectées. Par ailleurs, l'information donnée, tout particulièrement en maison d'hébergement, qui analyse la violence conjugale comme un problème social et qui cherche à développer un mouvement de solidarité entre les femmes, semble être un facteur important dans le processus d'empowerment identifié dans cette étude.

Nous croyons que l'utilisation du modèle du processus d'empowerment que nous avons élaboré peut être un apport intéressant en ce qui concerne l'intervention auprès des femmes victimes de violence conjugale. Le modèle permet d'identifier l'étape du processus à laquelle la victime se situe et les besoins qu'elle manifeste (émotifs, cognitifs, comportementaux). On pourra alors lui proposer un type d'aide et d'informations plus pertinent à ses besoins. L'utilisation de ce modèle offre aussi l'avantage de comprendre qu'il n'est peut-être pas le moment de proposer à une femme d'entreprendre une démarche légale et qu'elle n'est peut être pas prête à persévérer en ce sens.

ABSTRACT - While a significant number of studies on women victims of domestic violence have been conducted, studies which particularly concentrate on women whose cases came before the criminal justice system are rare. An examination of these cases allows us to identify of a variety of factors, particularly those which favour or inhibit recourse to criminal justice system. The most recent studies focus on the relationship between a woman's recourse to such proceedings and the process of her empowerment.

This article concerns a study specifically concerned with the empowerment process among women victims of domestic violence who had recourse to criminal justice process. Data for the study consisted of 29 semi-directive interviews with women victims, some who were and some who were not engaged in criminal justice process. Structural paradigm was used for data analysis. Three criteria prevailed in this choice, generally 
linked to definitions of violence toward women: a context of violence that involves a relationship of power characterized by dominance; attribution of the discriminative quality of the power relationship to gender membership; and recognition of the social and public dimensions of problems linked with this type of violence.

The empowerment model elaborated in this article seems appropriate to study the trajectory of women victims of domestic violence. It supports a hypothesis that every helping activity may potentially assist women victims' engagement in an empowerment process. The data also allows identification of elements which facilitate the process: emotional support and access to information. We were not able to identify elements specific to the criminal justice system, as a social institution, which would favour an empowerment process.

Each of the respondents identified both facilitating factors and obstacles to empowerment. Only the more social nature of their discourse distinguished respondents who completed the criminal justice process from those who did not. This article puts forward the hypothesis that completion of the criminal justice process is an indicator of empowerment. If this hypothesis is correct, it should nonetheless be concluded that a woman's decisions must be respected at every stage of the criminal justice process. In addition, access to information analyzing domestic violence as a social problem and aimed at the development of solidarity among women victims, particularly provided by shelter residence, was identified by this study as an important factor in the empowerment process.

The authors consider that the empowerment model elaborated in this article offers a perspective of interest for interventions aiming to assist women victims of domestic violence. The model defines the stages of the empowerment process, as well as the emotional, cognitive, and behavioural needs of women victims, making it possible to provide women with the types of assistance and information that are most relevant to them. The model further facilitates an understanding of whether criminal justice proceedings would be appropriate, and whether the woman victim will be able to persevere in this matter.

RESUMEN - Si bien el número de investigaciones realizadas sobre el tema de la mujer víctima de la violencia conyugal es importante a la par que diversificado, los trabajos específicos sobre las mujeres implicadas en el sistema judicial son hasta ahora escasos. Los estudios consultados han permitido - entre otras cosas - identificar los factores que facilitan la vía judicial y los factores que la frenan. Recientemente, otros estudios se han centrado en el análisis de las relaciones entre la posibilidad de una víctima de recurrir al sistema judicial y el mecanismo de obtención de poder legal o empowerment. El presente articulo tiene por objeto el interés particular por el proceso de empowerment en el caso de mujeres víctimas de violencia conyugal que han recurrido al sistema judicial. El mismo se apoya sobre el análisis de 29 entrevistas semi-dirigidas realizadas con mujeres víctimas de violencia conyugal, implicadas o no en un proceso judicial. El paradigma retenido para el análisis de los datos corresponde al paradigma estructural. En la selección de datos, prevalecieron tres criterios generalmente ligados a la definición de la violencia ejercida sobre la mujer, a saber : la violencia se ubica en un contexto de relaciones de poder caracterizado por la dominación ; el carácter discriminante de estas relaciones de poder se atribuye al hecho de pertenecer a un sexo más 
que al otro ; el reconocimiento de la dimensión social y pública del problema de la violencia.

El modelo de empowerment tal como lo hemos elaborado parece adecuado para el estudio de trayectorias en mujeres víctimas de violencia conyugal y su uso permite indicar que cualquier gestión de ayuda puede efectivamente facilitar la implicación de la mujer en el proceso de obtención de poder legal. Los datos recogidos permiten igualmente identificar los elementos que facilitan este proceso : apoyo emocional o apoyo informativo. No nos ha sido posible hasta ahora identificar los elementos específicos del sistema judicial, en cuanto institución social, que favorecen el proceso de empowerment. Todas las mujeres que respondieron identificaron los factores facilitantes, así como los obstáculos. En las respuestas sólo el discurso de naturaleza social permitió distinguir las mujeres que han completado el recorrido en el sistema judicial del resto de ellas. La hipótesis que retenemos hasta ahora es la de que el hecho de llevar a cabo las diligencias judiciales constituye más bien un indicador de empowerment. De ser ello cierto, podríamos concluir que cualesquiera sean las decisiones tomadas por las mujeres en las distintas etapas del proceso judicial, éstas deben ser respetadas. Por otra parte, la información que les es suministrada, en especial en las residencias para mujeres victimizadas y que analiza la violencia femenina como un problema social, procurando desarrollar así un movimiento de solidaridad entre las personas del sexo femenino, parece constituir un factor importante en el proceso de adquisición de poder legal identificado en el presente estudio.

Estamos convencidos de que la utilización del modelo de análisis del proceso de empowerment puede representar una contribución interesante en lo que se refiere a la intervención en el caso de mujeres víctimas de la violencia conyugal. El modelo permite identificar la etapa del proceso en la cual se sitúa la víctima, así como el tipo de necesidades que ella experimenta (emotivas, cognoscitivas, conductistas). De esta manera, podría proponerse la modalidad de ayuda y de información más pertinente en función de sus necesidades. La utilización de este modelo ofrece igualmente la ventaja de comprender cuando no es el momento oportuno para proponer a la mujer su implicación en un proceso legal, explicando porqué en ese momento no se halla tal vez en condiciones de perseverar en tal sentido.

\section{Contexte de l'étude}

L'étude qui est l'objet du présent article est issue d'une collaboration entre l'équipe VICTOIRE du CRI-VIFF, le Centre de Santé publique de Québec, la Maison des femmes de Québec et le Regroupement provincial des maisons d'hébergement et de transition pour femmes victimes de violence conjugale. Ces partenaires, provenant des secteurs communautaire, public et universitaire ainsi que de disciplines variées (droit, santé publique, travail social), se sont interrogés sur le vécu des victimes de violence conjugale à travers le système judiciaire. L'objectif de cette recherche était d'élaborer de nouvelles perspectives théoriques et pratiques en matière de violence conjugale et de contribuer à l'amélio- 
ration des politiques en la matière. Ce projet s'est intéressé de façon particulière au processus d'empowerment de femmes victimes de violence conjugale qui ont eu recours au système judiciaire. Le concept central retenu pour l'étude de notre problématique est le concept d'empowerment qui permet de décrire et de comprendre le processus par lequel les femmes traversent le système judiciaire.

L'étude comporte deux volets : 1) la description et la représentation statistique des plaintes relatives à la violence conjugale adressées à la Sûreté municipale de Québec en 1996 et, 2) l'exploration des trajectoires de femmes victimes de violence conjugale engagées dans un processus judiciaire, à la lumière du concept d'empowerment. Le présent article fait état de l'analyse préliminaire des résultats du deuxième volet de l'étude. Il porte sur l'analyse des données qualitatives se rapportant aux trajectoires de 29 femmes victimes de violence conjugale, engagées ou non dans un processus judiciaire, données recueillies dans le cadre d'entrevues semi-dirigées.

\section{Problématique}

En 1985 , le ministère de la Santé et des Services sociaux publie une première version de sa « Politique d'aide aux femmes violentées ». Un an plus tard, à l'instar des autres provinces canadiennes, le ministère de la Justice du Québec, en collaboration avec le Solliciteur général, adoptait la « Politique d'intervention en matière de violence conjugale ». Cette politique visait à judiciariser les infractions criminelles commises dans un contexte de violence conjugale. En 1995, le Québec réaffirme son engagement à agir pour contrer la violence conjugale à travers sa politique interministérielle d'intervention en matière de violence conjugale, intitulée "Prévenir, dépister et contrer la violence conjugale ». Ainsi, moins de dix ans plus tard, la politique sectorielle devenait interministérielle. Malgré la reconnaissance politique du problème et les efforts concertés pour augmenter les déclarations des victimes aux forces policières et les mises en accusation, l'enquête nationale de Statistiques Canada sur la violence envers les femmes (Rodgers, 1994) rapporte que seulement $26 \%$ des victimes de violence conjugale ont déclaré ces épisodes de violence à la police et que, d'autre part, plus de la moitié $(52 \%)$ de celles qui n'ont pas déclaré ces épisodes ne jugeaient pas l'événement suffisamment important pour le faire.

Bien qu'un nombre important de recherches, pourtant diversifiées, ait été réalisé auprès de femmes victimes de violence conjugale, les études 
portant spécifiquement sur les femmes engagées dans le processus judiciaire se font plutôt rares. Les recherches consultées ont permis d'identifier entre autres des facteurs facilitant le recours au processus judiciaire et des facteurs le freinant. Des études récentes, généralement qualitatives, ont porté de façon plus particulière sur les liens pouvant exister entre le fait de s'engager dans une démarche judiciaire et le processus d'empowerment chez des femmes victimes de violence conjugale. Bien que plusieurs auteurs (Ford, 1991 ; Hart, 1995 ; Light et Rivkin, 1996 ; Baker, 1997) associent cette démarche au processus d'empowerment, plusieurs comportements identifiés comme étant des démarches contribuant à l'empowerment des femmes victimes de violence ou étant la manifestation de cet empowerment nous apparaissaient en contradiction avec notre compréhension de l'empowerment. Comment un même concept pouvait-il être utilisé pour caractériser des démarches ayant pourtant des orientations qui semblent si différentes sinon diamétralement opposées? Afin de répondre à cette question et nous situer au moment de l'analyse de nos données, nous avons recensé les éléments de définition de ce concept faisant l'objet d'un consensus général dans la littérature. Nous avons ensuite examiné l'ensemble des critiques suscitées par ce concept, des limites et des enjeux identifiés. Trois paradigmes, implicites dans différents écrits portant sur l'empowerment, ont ainsi pu être dégagés : le paradigme technocratique, le paradigme structurel et le paradigme écologique. Bien sûr, l'adhésion à l'un ou l'autre de ces paradigmes « [...] influence la conception même du processus d'empowerment, la façon de poser les problèmes, la conception du changement social, le choix des méthodes de recherche [et d'intervention] et les objectifs poursuivis » (Dallaire et Chamberland, 1996 : 92).

Qu'est-ce au juste que l'empowerment? Au-delà des confusions apportées par cette première différence au niveau de sa définition, le terme est largement utilisé, particulièrement depuis une dizaine d'années, mais sa définition, sa conceptualisation et son utilisation ne font pas encore l'unanimité (Sheilds, 1995) et sont régulièrement soumises à la critique. Certaines définitions réfèrent au processus d'empowerment alors que d'autres définitions sont davantage centrées sur ses résultats (Zimmerman, 1995), et que d'autres enfin l'identifient à une approche d'intervention (Ninacs, 1995). Nonobstant ces différences, c'est le plus souvent à partir de la définition de Rappaport (1987) que les auteurs tentent de formuler une définition de l'empowerment. Pour Rappaport l'empowerment constitue « a mecanism by which people, organiza- 
tions and communities gain mastery over their affairs $»($ p. 122). L'exercice d'un tel contrôle demande l'accès à des ressources aussi bien matérielles, psychologiques que sociales. C'est pourquoi un consensus s'est établi progressivement autour de l'idée d'un progrès social destiné à augmenter l'accès aux ressources et aux opportunités des personnes démunies, au moyen d'une attitude pro-active de la part de celles-ci. L'accès aux ressources implique le développement d'une conscience critique de l'environnement et des rapports de pouvoir qui s'y déploient. Aussi, c'est généralement autour de l'idée de conscientisation telle que défendue par Freire (1977), c'est-à-dire une prise de conscience par l'action, que certains auteurs (Le Bossé et Lavallée, 1993 ; Breton, 1994 ; Lee, 1994 ; Parsons, 1995) définissent l'empowerment.

On constate donc plusieurs différences au plan de la conceptualisation de l'empowerment. Malgré ces différences, quatre aspects de l'empowerment ressortent de la documentation consultée et semblent rallier l'assentiment général. Tout d'abord, le gain de pouvoir constitue l'élément central de l'empowerment (Breton, 1993 ; Jutras, 1996). Ceci implique, de façon implicite ou explicite, qu'une certaine forme de déficit de pouvoir, réel ou perçu comme tel (Ninacs, 1995), est reconnue et qu'elle légitime la quête d'un gain. (Fait à noter, le concept de pouvoir, central au concept d'em-power-ment, n'est habituellement pas défini par les auteurs.) Deuxièmement, il y a consensus chez la plupart des auteurs que l'initiation du processus d'empowerment est autodéterminée. Troisièmement, l'empowerment est intrinsèquement associé à l'action (Rappaport, 1987 ; Breton, 1993 ; Le Bossé et Lavallée, 1993 ; Riger, 1993 ; Perkins et Zimmerman, 1995 ; Rodwell, 1996 ; Le Bossé, 1996). Les actions qui sont entreprises par les personnes elles-mêmes concourent au processus d'empowerment, tandis que leurs bénéfices correspondent au résultat concret de celui-ci. Enfin, le concept d'empowerment est multidimensionnel. Le gain de pouvoir peut être recherché dans plusieurs dimensions de la vie (personnelle, interpersonnelle, sociale et collective) et ce, tant sur le plan subjectif que sur celui des conditions objectives. Considérer l'une ou l'autre de ces dimensions c'est faire appel à des grilles d'analyse et à des disciplines différentes, particulièrement à la psychologie et à la sociologie (Rappaport, 1985 ; Swift et Levine, 1987 ; Breton, 1994 ; Zimmerman, 1995). Ces grilles reposent sur des paradigmes précis dont les composantes déterminent la façon de définir les problèmes et leur origine, individu (psychologie) ou société (sociologie), les changements souhaités (individuels et/ou collectifs) et les 
moyens à privilégier. C'est précisément l'analyse de ces composantes qui a présidé à l'élaboration de la typologie des paradigmes de l'empowerment présentée ci-après.

Le paradigme nommé technocratique (peu utilisé) est fortement influencé par la psychologie. On y attribue le déficit de pouvoir à des déficits personnels exclusivement, à la méconnaissance qu'ont les individus de leurs propres capacités ou compétences et à la sous-utilisation de celles-ci (Jutras, 1996 ; Le Bossé, 1996 ; Drolet, 1997). On s’intéresse donc aux incapacités individuelles. Modifier les perceptions et les comportements individuels de façon à responsabiliser les personnes et à faire en sorte qu'elles soient capables de résoudre elles-mêmes leurs problèmes personnels et de relever les défis qu'elles rencontrent, voilà la cible du changement envisagé. L'empowerment se situe au niveau individuel seulement et se traduit par l'augmentation de l'estime de soi, de la motivation et du sens des responsabilités des individus (Conger et Kanungo, 1988) moyennant le transfert ou le partage de pouvoir (Rodwell, 1996). Ce paradigme s'inscrit dans le courant de l'individualisme méthodologique et est surtout inspiré des idéologies organisationnelles ayant un caractère plutôt hiérarchisé et patriarcal (Rodwell, 1996).

Le paradigme écologique quant à lui tient compte à la fois des individus et du contexte à l'intérieur duquel ils se situent (Rappaport, 1987 ; Le Bossé, 1995 ; Zimmerman, 1995). On s'intéresse ici aux conditions « incapacitantes » relatives aux individus. Bien que les rapports sociaux soient perçus comme inéquitables et discriminatoires et que l'impact des macro-systèmes tant sur les individus, que sur les familles et les communautés soit reconnu, on cherche davantage à en diminuer l'impact négatif qu'à les transformer. Le changement ciblé consiste à habiliter l'individu à comprendre ce qui lui arrive, à accéder à ses ressources personnelles et à agir dans un contexte précis. Le changement social peut également se produire, prendre plusieurs formes et résulter non pas d'une action conflictuelle mais plutôt consensuelle (Zimmerman, 1995). Le gain de pouvoir envisagé vise à récupérer un pouvoir d'influence nécessaire pour négocier la restauration de rapports sociaux équitables (Zimmerman, 1995 ; Dallaire et Chamberland, 1996 ; Le Bossé, 1996 ; Drolet, 1997). L'empowerment référerait ici à l'opportunité d'agir plus librement dans certains domaines ou contextes à partir d'habiletés particulières permettant de partager ou de renégocier le pouvoir (Riger, 1993). Ce paradigme rejoint le modèle de la psychologie communau- 
taire, lequel est inspiré fortement par les idéologies psychologiques et environnementales (Rodwell, 1996).

À l'opposé du paradigme technocratique, le paradigme structurel, plus proche de la sociologie critique et de la politique, sert également de toile de fond aux efforts de conceptualisation de nombreux auteurs qui se sont intéressés à l'empowerment. Le paradigme structurel identifie le manque de pouvoir à une domination résultant d'arrangements sociaux inéquitables et à des forces sociales répressives plutôt qu'à des déficits personnels (Dallaire et Chamberland, 1996: 129. cité par Lord et Dufort, 1996: 13 [traduction française]). Selon le paradigme structurel, le pouvoir est inscrit dans des rapports sociaux qui sont inégaux, « caractérisés par la domination, la subordination et la résistance, où les personnes [ou groupes] dominantes exercent leur pouvoir en limitant l'accès aux ressources matérielles et en inspirant aux personnes [ou groupes] la peur ou en leur inculquant des opinions auto-dévalorisantes à leur sujet » (Prilleltensky et Gonick, 1996: 129 cité par Lord et Dufort, 1996 : 13 [traduction française]). Cette dernière conception suscite une approche plus conflictuelle des solutions et vise la transformation des structures sociales qui créent et maintiennent les inégalités sociales. L'empowerment devient ainsi politique en soi (Drolet, 1997), passe par un pouvoir formel et se traduit par l'appropriation d'un pouvoir sur les dimensions socio-politiques qui affectent la vie des individus et limitent leur accès aux ressources et aux opportunités. Ce paradigme est surtout inspiré par les idéologies socio-politiques et féministes défendant des valeurs démocratiques fondées sur des rapports égalitaires Rodwell, 1996).

Le paradigme que nous avons retenu pour analyser le processus d'empowerment des femmes victimes de violence conjugale dans le système judiciaire est le paradigme structurel. Trois caractéristiques généralement liées à la définition de la violence faite aux femmes ont dirigé ce choix ;

- cette violence est située dans un contexte de relations de pouvoir caractérisées par la domination ;

- le caractère discriminant de ces relations de pouvoir est lié au fait d'appartenir à un sexe plutôt qu'à un autre ;

- la dimension sociale et publique du problème de cette violence est reconnue ; ceci tient compte de la contribution de systèmes plus larges à l'existence du phénomène de la violence conjugale. 
Ces éléments de définition évitent de considérer l'expérience de violence conjugale des femmes à partir des seuls facteurs intrapsychiques ou pathologiques et permettent de relativiser leur importance sans toutefois les exclure.

\section{Modèle théorique du processus d'empowerment}

Rappelons qu'un des objectifs de notre recherche est l'exploration de parcours de femmes victimes de violence conjugale à travers le système judiciaire et que nous avons porté un intérêt particulier à l'aspect dynamique et progressif du concept d'empowerment. Le processus d'empowerment est approprié pour une étude sur la violence conjugale dans la mesure où il permet de tenir compte de la dimension individuelle du phénomène vécu, qu'il la dépasse pour englober une dimension sociale et qu'il mène à une action. L'éventail des dimensions touchées par le processus d'empowerment favorise ainsi l'accès aux conditions structurelles du phénomène de la violence conjugale.

Pour des fins d'analyse, un modèle ouvert du processus d'empowerment a été conçu et ses divers éléments ont servi à élaborer les premiers axes autour desquels s'est articulée l'analyse des données. Cette modélisation est présentée schématiquement à l'aide d'un tableau (tableau 1). Le processus d'empowerment serait constitué de trois étapes : le déficit de pouvoir, la prise de conscience et le gain de pouvoir. À chacune de ces étapes, il a été possible d'identifier des émotions, des cognitions et des comportements qui y sont associés. Spécifions, encore une fois, que le processus d'empowerment est dynamique alors que ce tableau est linéaire, ce qui ne rend pas compte de la valeur de ce concept, et qu'il s'agit ici d'une synthèse de l'ensemble des caractéristiques retenues plutôt que du portrait de la dynamique du processus lui-même.

\section{Résultats}

Des intervenantes et intervenants de divers organismes (communautaires, judiciaires et institutionnels) ont contribué à identifier 48 informatrices potentielles. Les critères ayant présidé à la sélection des participantes étaient les suivants : réunir un nombre proportionnel d'informatrices ayant parcouru trois trajectoires types, soit ayant 


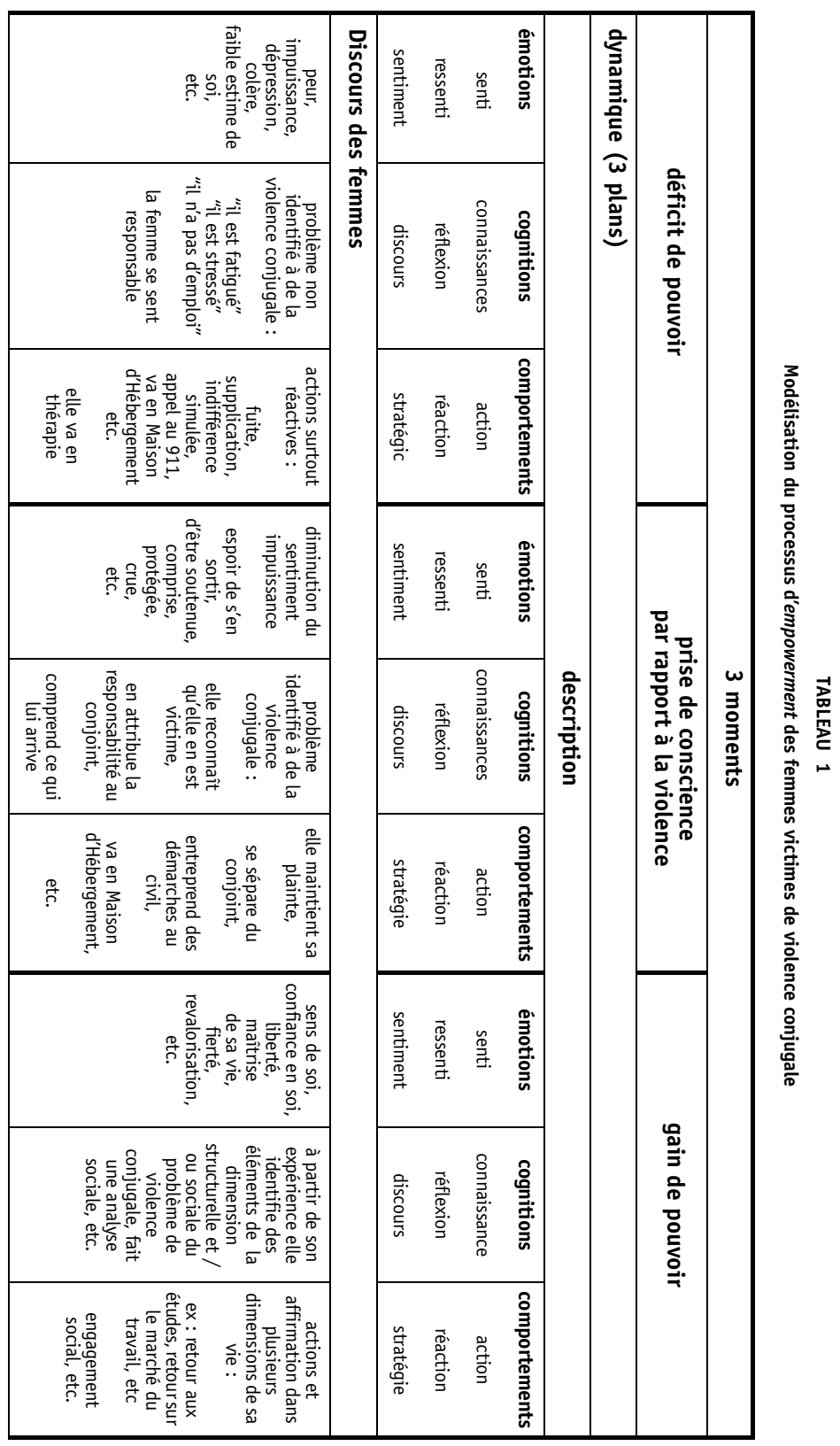


complété le processus judiciaire, ayant interrompu leur participation aux démarches judiciaires et n'ayant effectué aucune démarche judiciaire. En tout, 29 informatrices de la région de Québec ont été rencontrées : 14 femmes avaient complété le processus judiciaire, 7 l'avaient interrompu et 8 n'avaient jamais fait de signalement aux forces policières.

\section{Profil des informatrices}

La majorité des informatrices rencontrées, soit 23 sur 29, étaient âgées entre 30 et 49 ans au moment de l'entrevue, 7 d'entre elles appartenaient au groupe d'âge des 30-39 ans et 16 femmes avaient entre 40 et 49 ans. Les six autres informatrices se répartissaient, dans une proportion égale, entre les trois groupes d'âge suivants : 20-29 ans, 50-59 ans et 60 ans ou plus. Au moment de l'entrevue, presque toutes les informatrices, soit 27, habitaient seules, l'une habitait toujours avec son conjoint, alors qu'une autre a indiqué vivre avec son conjoint de temps à autre. Le nombre d'années d'études complétées par les informatrices est plutôt varié. Six (6) d'entre elles avaient complété le primaire, 8 le secondaire, 11 avaient terminé des études collégiales et 4 autres informatrices avaient achevé des études universitaires. Au moment de l'entrevue, 14 femmes occupaient un emploi, à temps plein ou à temps partiel, et 10 étaient sans emploi, recevant des prestations de la sécurité du revenu, de l'assurance-emploi ou de l'assurance-maladie. Cinq (5) informatrices poursuivaient des études. Toutes les femmes rencontrées vivaient de leurs propres revenus et l'une d'elles bénéficiait d'un revenu additionnel provenant de l'ex-conjoint. Un tiers des informatrices avaient un revenu annuel inférieur à 10000 \$. La majorité possédaient un revenu annuel se situant entre 10000 \$ et 30000 \$. Seulement quatre informatrices jouissaient d'un revenu annuel supérieur à 30000 \$. Plus des trois quarts des informatrices, soit 25 femmes sur 29 avaient des enfants, et de ce nombre, 18 avaient des enfants d'âge mineur. Parmi ces dernières, seulement deux n'en n'avaient pas ou ne partageaient pas la garde légale des enfants.

Lorsque les informatrices ont décrit les formes de violence dont elles avaient été victimes durant la relation avec le conjoint violent, toutes ont indiqué avoir subi de la violence psychologique et verbale à maintes reprises. À l'exception d'une seule informatrice, toutes ont rapporté des agressions physiques, contre leurs biens ou contre leur personne. Plus $\mathrm{du}$ tiers des informatrices ont confié avoir été victimes de violence sexuelle $(37,9 \%)$ tandis que plus de la moitié ont parlé de violence économique $(55,2 \%)$. Pour la majorité des informatrices rencontrées, la violence 
conjugale s'est poursuivie également après la rupture d'avec leur conjoint violent. Il s'agissait alors de violence psychologique et verbale, physique et économique. La violence psychologique et verbale s'est traduite par une manipulation au travers des enfants (aliénation parentale, garde légale, choix de garderie), des menaces de toute nature, du harcèlement ou encore, pour une informatrice, par le dévoilement public de souvenirs pénibles liés à son passé personnel. La violence physique exercée à l'endroit des femmes elles-mêmes consistait en des agressions de la part de l'ex-conjoint violent ou commandées par celui-ci à une tierce personne. Les biens de certaines femmes ont également été la cible de vandalisme, d'incendies. Certaines rapportaient aussi l'entrée par effraction dans leur domicile. Enfin, la violence économique rapportée résidait dans le fait de ne pas donner à l'informatrice l'accès à ses biens, de les détruire, de les vendre ou dans le défaut de payer la pension alimentaire ou de respecter les engagements pris en cette matière.

\section{Démarches effectuées}

Presque toutes les informatrices ont fait appel à des ressources communautaires telles que les maisons d'hébergement, les maisons de la famille, les groupes de femmes, Viol-secours, CAVAC (Centre d'aide aux victimes d'actes criminels), etc. Tant les informatrices qui ont passé au travers toutes les étapes du processus judiciaire que celles qui ne s'y sont pas engagées du tout ont rapporté des démarches effectuées auprès et/ ou avec de telles ressources, dans une proportion de $90 \%$. Plus des deux tiers d'entre elles ont fait appel à des ressources psychosociales (CLSC, thérapies, programmes d'aide aux employés, etc.) ou du milieu de la santé (médecins, infirmières, etc.) et $41 \%$ des informatrices ont fait appel à des ressources alternatives (groupes d'entraide, médecines douces, centres de désintoxication, etc.). Parallèlement à ces démarches, plus de la moitié des informatrices ont rapporté avoir entrepris des procédures judiciaires au civil (divorce, garde d'enfant, etc.), dans des proportions de $71 \%$ pour celles ayant complété le processus judiciaire, de $50 \%$ pour celles ayant interrompu ce processus et de $46 \%$ pour les femmes n'ayant intenté aucune procédure judiciaire au criminel.

\section{Analyse des données : première étape}

Notre première étape d'analyse a consisté à explorer le contenu des catégories préétablies correspondant au modèle précité, de façon à vérifier s'il existait des différences entre les discours des informatrices des 
trois cohortes. Rappelons que les résultats présentés ici sont préliminaires et non exhaustifs. Ainsi, nous avons constaté qu'il y avait des informatrices de chacune des cohortes pour presque toutes les catégories issues du modèle théorique. Cette observation signifie que ce modèle offre des caractéristiques appropriées pour décrire et analyser l'expérience d'empowerment de femmes victimes de violence conjugale. Par ailleurs, l'analyse des propos des informatrices a révélé surtout des similitudes dont plusieurs mettent en évidence certains faits. D'autres ressemblances identifiées rejoignent, pour leur part, les conclusions d'études consultées portant sur cette problématique (notamment, l'utilisation du signalement pour récupérer du pouvoir face au conjoint violent et les facteurs aidants et non aidants pour l'amorce d'une démarche judiciaire).

C'est la violence physique qui amène le plus souvent les femmes à prendre conscience qu'elles sont victimes de violence conjugale. Plusieurs informatrices croyaient jusqu'alors qu'elles avaient un problème de relation conjugale et que leur sécurité n'était pas en jeu. Les propos de cette informatrice sont représentatifs à ce sujet :

J'étais consciente qu'il y avait quelque chose qui ne marchait pas, mais je n'étais pas consciente que c'était de la violence. Dans ma tête à moi, de la violence, il fallait que je sois à terre, en sang, avec un bras cassé, comme on voit à la télévision dans les émissions [sur le sujet]. Mais ce n'est pas ça, il devrait y avoir des émissions avec le début, car c'est au début qu'il faut agir, quand tu es rendue là il est trop tard. [...] le dénigrement, les insultes, les menaces... oui puis peut-être aussi le début de la violence physique, juste tasser dans un mur, juste même lancer un objet, c'est de la violence. Il faut agir là, parce qu'après il va être trop tard (Informatrice 25).

Ces propos, communs à plusieurs femmes, révèlent qu'elles ont intégré le discours social dénonçant la violence conjugale, que celle-ci est identifiée à une agression physique, laquelle est punissable par la loi. C'est le plus souvent un tel épisode de violence physique qui amène les femmes à consulter pour ensuite découvrir qu'il s'agit en fait d'une des nombreuses formes (psychologique et verbale, sexuelle, économique, etc.) de la violence conjugale, ces dernières formes étant peu souvent judiciarisées. C'est le plus souvent à partir de ce moment et à partir de l'intégration de l'analyse proposée par les intervenantes consultées (au CLSC, en maison d'hébergement), qu'elles cesseront de s'attribuer la responsabilité de la violence et l'attribueront désormais à leur conjoint. À titre d'illustration, mentionnons les propos de cette informatrice, qui a séjourné en maison d'hébergement : 
Je ne savais pas vraiment, avant d'arriver à la maison d'hébergement, quel style de violence je vivais mais ce que je réalisais, c'est qu'on était en train de me détruire. J'ai pris conscience aussi qu'en réalité, c'est pas moi qui étais malade, puis qui étais folle, puis qui étais sautée. J’ai pris conscience que j'avais été manipulée. La personne responsable c'est lui (Informatrice 17).

Comparativement aux informatrices qui n'ont pas effectué de démarche de demande d'aide, celles qui ont séjourné en maison d'hébergement et/ou qui ont entrepris une autre démarche de demande d'aide, ont acquis des connaissances plus complètes sur les diverses formes de violence (physique, psychologique et verbale, sexuelle, économique), les cycles de la violence et la responsabilité du conjoint violent.

Cependant, on remarque un grand nombre de propos similaires chez les informatrices rencontrées. D'une part, c'est un épisode de violence physique qui a entraîné une prise de conscience de leur situation. D'autre part, toutes avaient intégré le fait, socialement reconnu, que cette violence est inacceptable. Par contre, les femmes qui ont réalisé une démarche de soutien jouissent de connaissances plus complètes sur la violence vécue, sur les nombreux éléments qui constituent le phénomène de la violence conjugale, sont mieux outillées pour poursuivre les démarches subséquentes à cette expérience et leur compréhension des rapports femmes/ hommes est plus globale. En résumé, la prise de conscience s'effectue souvent au moment d'une situation de violence physique, associée à une information obtenue en maison d'hébergement ou par l'entremise d'autres intervenant-e-s du système judiciaire ou du système institutionnel. Quelles que soient les cohortes, les raisons de faire appel au système judiciaire sont les mêmes, les facteurs aidants et les obstacles influant sur la poursuite de cette démarche légale aussi, qu'il s'agisse d'aspects relevant du système judiciaire lui-même ou de ses acteurs, ou encore de l'agresseur (risque de récidive et de représailles) et de la victime (facteurs personnels, économiques ou liés à son environnement).

Puisque cette première étape n'a pas permis d'identifier de différences entre les discours des informatrices de chacune des cohortes, en ce qui a trait aux différentes catégories issues du modèle théorique, nous avons tenté d'analyser les discours qui cumulaient le plus grand nombre de caractéristiques du troisième moment du processus d'empowerment (les gains et le développement de préoccupations sociales) ; en somme nous nous sommes demandé si le discours des informatrices qui se situaient le plus dans la troisième étape du processus d'empowerment avait des caractéristiques particulières. 


\section{Analyse des données : deuxième étape}

Cette opération a effectivement permis d'identifier des différences entre les discours des informatrices selon la cohorte à laquelle elles appartenaient. La première différence concernait la composition du groupe d'informatrices qui cumulaient le plus de caractéristiques correspondant au moment 3 du processus d'empowerment. Rappelons que ce moment est associé à un dépassement de l'analyse individuelle du problème vers une analyse sociale et l'émergence d'un mouvement de solidarité à l'égard des autres victimes de violence conjugale. Nous avons ainsi identifié 7 informatrices ayant complété le processus judiciaire (sur une possibilité de 14) et 2 informatrices n'ayant effectué aucune démarche judiciaire (dont une qui prévoit le faire dès qu'elle disposera de suffisamment de preuves) faisant montre de ces préoccupations. Aucune des informatrices ayant interrompu leur participation au processus judiciaire ne figure au nombre de celles qui cumulent le plus de caractéristiques correspondant au troisième moment $\mathrm{du}$ processus. C'est pour cette raison que nous avons décidé d'explorer et de comparer le discours de ces informatrices avec celui de celles réunissant plusieurs de ces caractéristiques. Ce sont ces résultats préliminaires que nous présentons ici.

\section{Signalement}

Déjà, au sujet de la toute première étape des procédures judiciaires, le discours des informatrices qui ont complété ces procédures se distingue de celui des autres femmes rencontrées. En effet, leur discours s'avère plus affirmatif, alors que les propos de celles qui ont interrompu leurs démarches est plus réactif. Les raisons mentionnées par la première catégorie d'informatrices pour expliquer pourquoi elles ont persisté jusqu'au bout des procédures judiciaires sont les suivantes : trois informatrices veulent dénoncer la violence qu'elles ont elles-mêmes subie et la violence conjugale en général, deux souhaitent retrouver leur autonomie ou reprendre leur pouvoir au conjoint violent, une autre désire « grandir » et, finalement, une dernière a peur pour sa sécurité bien qu'elle ait fait appel, à plusieurs reprises, au système judiciaire pour mettre fin au harcèlement et à la violence (menaces, chantage, incendie) de l'ex-conjoint violent.

Parmi celles qui ont décidé de ne pas continuer les procédures judiciaires, par exemple en refusant de témoigner à l'enquête préliminaire, amenant ainsi une fin aux procédures faute de preuve, nous avons identifié des éléments de type plutôt réactif. Pour une informatrice, 
l'appréhension de ne pas être crue a été le motif de son choix ; pour deux autres, il s'agissait de compassion envers le conjoint ou d'un souci pour les enfants, une troisième s'inquiétait des impacts financiers éventuels, ne jouissant pas elle-même d'une autonomie financière, une autre craignait pour sa vie et, finalement, une dernière voulait faire peur au conjoint par un appel à la police sans souhaiter aller au-delà de cette première étape.

Du côté de celles qui n'ont effectué aucune démarche judiciaire et qui présentent les caractéristiques du moment 3 (2 femmes), une seule fournit de l'information sur cet aspect en disant qu'elle attend d'avoir assez de preuves avant de mettre en branle des démarches judiciaires, alors que la deuxième le passe sous silence.

\section{Élément déclencheur}

L'élément déclencheur de la prise de conscience d'être victime de violence conjugale est le degré de dangerosité pour elle-même, pour les enfants ou pour les proches, rendu manifeste à la suite d'un épisode de violence physique, de menaces de mort ou d'une démarche pour solliciter de l'aide. Pour les informatrices qui ont maintenu leur démarche auprès du système judiciaire, ces événements ont favorisé leur prise de conscience en ce qui a trait à leur situation et leur ont permis d'entreprendre mais surtout de poursuivre les procédures judiciaires.

Quant aux informatrices qui ont fait appel au 9-1-1 mais qui n'ont pas opté pour l'utilisation du système judiciaire, trois ont identifié leur situation de violence conjugale suite à une scène de violence physique. Trois autres informatrices ont pris conscience de leur situation de violence conjugale auprès de ressources consultées alors qu'une dernière a associé cette prise de conscience à sa sobriété (elle est maintenant une alcoolique sobre).

\section{Bilan sur le système judiciaire}

Quel bilan les informatrices se retrouvant à la dernière étape du processus d'empowerment font-elles du système judiciaire? Quels sont les émotions, les cognitions et les comportements qu'elles nous expriment? Sur les sept informatrices se situant à ce moment du processus et ayant maintenu leur participation à travers toutes les étapes du processus judiciaire, trois considèrent qu'elles ne se sentent ou ne se sont pas senties assez protégées du conjoint violent. Trois informatrices mentionnent qu'elles considèrent que les démarches judiciaires ne sont pas assez 
rapides, ce qui constituait un danger pour elles. En ce qui concerne les peines imposées au conjoint violent, quatre jugent qu'elles ne sont pas assez sévères et souvent plus légères que lorsqu'il s'agit de violence générale (non conjugale). Pour ce qui est du système judiciaire en tant que tel, ou de ses acteurs, nos informatrices l'ont trouvé lourd, « violent » pour une femme qui porte plainte; quatre d'entre elles ont souligné qu'elles avaient eu l'impression qu'on faisait leur procès à elles et une autre a senti qu'elle était manipulée dans ces démarches.

Si l'on se penche sur les raisons évoquées par les femmes qui ont décidé de ne pas entreprendre de démarches légales, une informatrice a insisté sur le fait qu'elle ne faisait pas confiance au système judiciaire et que la violence conjugale n'en constituait pas une priorité. Une autre a mentionné qu'elle ne se sentait pas protégée ; trois considèrent que ces démarches légales ne sont pas assez rapides et que le danger pour les femmes demeure important. Encore une fois, une informatrice trouve que les peines infligées aux conjoints violents ne sont pas assez sévères et qu'elles s'avèrent non proportionnelles aux efforts investis par les femmes. L'impression que le procès intenté par une femme qui porte plainte pour violence conjugale devient son procès à elle a de nouveau été relevée par deux informatrices et une autre a mentionné l'absence de contrôle sur les hommes accusés de violence conjugale.

\section{Préoccupations sociales}

À l'exception d'une informatrice, toutes celles qui avaient complété les procédures judiciaires ont exprimé une préoccupation sociale positive à l'égard des autres femmes, par exemple en spécifiant vouloir éviter à d'autres femmes de connaître le même sort qu'elles, pour cinq d'entre elles, ou en souhaitant aider d'autres femmes en montrant qu'il est possible de s'en sortir. Deux autres œuvrent dans leur milieu de travail à sensibiliser les gens au problème des femmes victimes de violence conjugale. Une informatrice a cependant indiqué s'être sentie l'objet de manipulations pour la cause des femmes durant ses démarches judiciaires.

En ce qui concerne celles qui n'ont pas voulu qu'il y ait poursuite du conjoint violent, trois informatrices seulement ont exprimé une préoccupation sociale à l'endroit des autres femmes victimes de violence conjugale, en aidant ou voulant aider, et en utilisant le signalement pour prévenir que le conjoint s'en prenne à une nouvelle conjointe. Une autre informatrice a tenu des propos que l'on pourrait qualifier d'individualistes, 
en soulignant qu'elle s'occupait d'elle-même et non des autres femmes, alors que les trois autres n'ont pas parlé de préoccupations sociales.

Facteurs aidants reliés

aux acteurs du système judiciaire

Selon les informatrices, le fait d'avoir été crues par divers acteurs du système judiciaire leur a permis de légitimer et de valider leur vécu, leur volonté de mettre fin à cette situation ainsi que les démarches qu'elles acceptaient de poursuivre. Par ailleurs, elles ont apprécié le fait qu'en plus de les croire, différents acteurs du système judiciaire les appuient dans leurs démarches, dénoncent la violence conjugale, leur fournissent les informations pertinentes et leur apportent le soutien nécessaire au fur et à mesure du déroulement des procédures. Par ces attitudes jugées positives par nos informatrices, elles se sont senties accueillies dans leurs demandes et respectées. Finalement, en ce qui concerne les informations obtenues, le fait de découvrir que la violence conjugale ne se limite pas uniquement à la violence physique, mais que plusieurs formes et dimensions la constituent, a favorisé l'émergence et le maintien de la force et du courage nécessaires à la poursuite de démarches judiciaires ${ }^{3}$.

Par contre, aucune des informatrices ayant décidé de retirer leur plainte initiale n'a mentionné d'éléments de ce discours sur les facteurs aidants même si la majorité d'entre elles ont rencontré des intervenant-e-s lors de leurs démarches de demande d'aide.

Ces commentaires nous permettent de voir que les facteurs aidants se retrouvent à la fois au niveau émotif, cognitif et comportemental. En effet, le soutien émotif, l'information reçue et les actions concrètes sont autant d'éléments qui ont permis aux femmes d'amorcer et de poursuivre des démarches au niveau judiciaire.

Obstacles reliés aux divers

acteurs du système judiciaire

Les informatrices ont également identifié des obstacles rencontrés lors de leurs démarches. Cinq informatrices ont affirmé qu'elles avaient l'impression qu'on les avait rendues responsables de la situation de violence dans laquelle elles étaient, dont trois informatrices à cause de leur propre pathologie (dépression et alcoolisme) ; une autre a senti

3. En plus, bien sûr, du soutien concret apporté par les différentes intervenantes des maisons d'hébergement ou du CAVAC par l'accompagnement en cour, par les informations données, etc. 
qu'on faisait reposer sur elle la responsabilité de maintenir l'union dans l'intérêt du développement psychologique harmonieux de l'enfant.

Le manque d'information a également été identifié comme un obstacle. Que ce soit l'absence d'information sur les autres formes de violence, sur leurs conséquences ou sur les ressources disponibles, les répondantes ont soutenu que ce manque constituait une lacune importante dans leur processus d'empowerment.

Enfin, plusieurs informatrices ont trouvé pénible le fait qu'une femme ne puisse solliciter de protection judiciaire ou entreprendre une démarche légale que si elle a été victime d'une agression physique grave. La difficulté à construire la preuve a également été mentionnée par plusieurs informatrices.

\section{Discussion}

Si l'on tient compte des deux étapes d'analyse effectuées, on peut affirmer que le modèle de l'empowerment tel que nous l'avions élaboré est approprié pour l'étude de trajectoires de femmes victimes de violence conjugale. En effet, toutes les répondantes, quelle que soit la cohorte, ont décrit des émotions, des cognitions et des comportements liés à chacun des trois moments du processus tel que nous l'avions élaboré (déficit de pouvoir, prise de conscience et gain de pouvoir). Ceci laisse supposer que toute démarche d'aide peut aider les femmes à s'engager dans un processus d'empowerment. Nos données nous permettent également d'identifier des éléments qui facilitent ce processus : support émotionnel, information sur les formes de violence, sur le cycle de la violence ou encore sur la responsabilité de celle-ci, et actions concrètes d'aide et d'accompagnement. Nous n'avons toutefois pas pu cerner des éléments spécifiques au système judiciaire, en tant qu'institution sociale, qui favoriseraient le processus d'empowerment. Toutes les répondantes ont identifié des facteurs aidants et des obstacles. Seule la nature plus sociale de leur discours différencie les répondantes qui ont complété le parcours dans le système judiciaire des autres répondantes. L'hypothèse que nous retenons en ce moment est que le fait de mener à terme des démarches judiciaires est plutôt indicateur d'empowerment. Si ceci s'avérait juste, on devrait en conclure que les femmes qui entreprennent et maintiennent des démarches judiciaires sont celles qui sont les plus armées pour le faire. En corollaire, quelles que soient les décisions prises par les femmes à chacune des étapes du processus judiciaire, ces décisions doivent être respectées. Une femme qui 
n'est pas prête à entreprendre des démarches devrait être respectée dans ce choix. Par ailleurs, l'information donnée, tout particulièrement en maison d'hébergement, qui analyse la violence conjugale comme un problème social et qui cherche à développer un mouvement de solidarité, semble être un facteur important dans le processus d'empowerment.

Nous croyons que l'utilisation du modèle du processus d'empowerment peut être un apport intéressant en ce qui concerne l'intervention auprès des femmes victimes de violence conjugale. Le modèle permet d'identifier l'étape du processus à laquelle la victime se situe, les besoins qu'elle manifeste (émotifs, cognitifs, comportementaux). On pourra alors lui proposer un type d'aide et d'informations plus pertinent à ses besoins. L'utilisation de ce modèle offre aussi l'avantage de comprendre qu'il n'est peut-être pas le moment de proposer à une femme d'entreprendre une démarche légale et qu'elle n'est peut-être pas prête à persévérer en ce sens.

\section{Conclusion}

Toutes les informatrices rencontrées ont sollicité une forme d'aide à l'extérieur de leur environnement immédiat (maison d'hébergement, CLSC, 9-1-1, etc.), ce qui les a menées à un changement de perspective sur leur situation. Trois cohortes ont pu être constituées autour de trois parcours types : les femmes victimes de violence conjugale qui ne se sont pas engagées dans le processus judiciaire ; celles qui s'y sont engagées pour ensuite s'en retirer ; et celles qui s'y sont engagées et ont poursuivi cette démarche jusqu'au bout. Les différentes caractéristiques du modèle du processus d'empowerment ont présidé à l'élaboration de notre grille de codification.

Il est ainsi possible de confirmer que les parcours des informatrices rencontrées possèdent toutes des caractéristiques du modèle élaboré. Cette observation préliminaire tend à confirmer que notre modèle apparaît approprié pour étudier le processus d'empowerment des femmes dans le champ de la violence conjugale, à tout le moins pour celles qui ont sollicité une forme ou une autre d'aide. Toujours à titre d'observation préliminaire, notons que la prise en compte des perceptions des informatrices par rapport aux différents acteurs côtoyés offre l'avantage d'identifier les différents facteurs contribuant à leur processus d'empowerment et qui s'inscrivent au-delà de la réalité individuelle des victimes. Cet exercice, en plus de mettre en évidence des conditions objectives, 
permet de repérer les éléments de la dynamique collective qui contribuent à ce processus, à savoir : les valeurs sociales, les cognitions (information, discours) et les comportements (actions, interventions, etc.). Ces informations peuvent offrir des pistes intéressantes pour l'évaluation des différentes solutions et ressources offertes aux femmes victimes de violence conjugale et qui visent leur autonomie.

\section{Références}

BAKER, P. L. 1997. «And I went back. Battered women's negotiation of choice ", Journal of Contemporary Ethnography 26 (1) : 55-74.

BRETON, M. 1993. "On the meaning of empowerment and empowermentoriented social work practice », texte présenté à la séance plénière du $\mathrm{XV}^{e}$ Symposium annuel de l'«Association for the Advancement of Social Work with Groups ». New York.

BRETON, M. 1994. «Relating Competence-Promotion and Empowerment», Journal of Progressive Human Services 5 (1) : 27-44.

CONGER, J. A. et KANUNGO, R. N. 1988. « The Empowerment Process: Integration Theory and Practice», Academy of Management Review 13 (3) : 471-482.

Dallaire, N. et Chamberland, C. 1996. «Empowerment, crises et modernité », Revue canadienne de santé mentale communautaire 15 (2) : 87-107.

Drolet, M. 1997. "L'empowerment et intervention familiale : concept paradoxal occultant parfois la pauvreté », Reflets 3 (1) : 55-79.

FORD, D. A. 1991. «Prosecution as a Victim Power Resource: A Note on Empowering Women in Violent Conjugal Relationship », Law and Society Review 25 (2) : 313-334.

FreIRE, P. 1977. Pédagogie des opprimés, Paris : Petite collection Maspero.

GOUVERNEMENT DU QUÉBEC 1995. Prévenir, dépister, contrer la violence. politique d'intervention en matière de violence conjugale, Québec: Bibliothèque nationale du Québec.

HART, B. 1995. «Battered Women in the Criminal Justice System », American Behavioral Scientist 36 (5) : 624-638.

JUTRAS, S. 1996. «L'appropriation. Un modèle approprié pour la promotion de la santé mentale des enfants? » Revue canadienne de santé mentale communautaire 15 (2) : 123-142.

LE BOSSÉ, Y. 1995. Étude exploratoire du phénomène de l'empowerment, thèse de doctorat déposée à la Faculté des études graduées de l'Université Laval. Québec : Université Laval.

LE BOSSÉ, Y. 1996. «Empowerment et pratiques sociales : illustration d'une utopie prise au sérieux ", Nouvelles pratiques sociales 9 (1) : 127-145.

LE Bossé, Y. et LAVAllÉE, M. C. 1993. «Empowerment et psychologie communautaire: aperçu historique et perspectives d'avenir », Cahiers internationaux de psychologie sociale 18 (2) : 7-20.

LEE, J. J. A. B. 1994. The empowerment approach to social work practice, New York : Columbia University Press. 
LIGHT, L. et RivkiN, S. 1996. « Le pouvoir, la domination et la violence dans les relations familiales : réponses du système de justice », p. 185-195 in Mettre fin à la violence: La famille se transforme, le futur se forme, Institut de la Colombie-Britannique sur la violence familiale. Colombie-Britannique : Benwell Atkins limited.

LORD, J. et DUFORT, F. 1996. « Le pouvoir et l'oppression en santé mentale », Revue canadienne de santé mentale communautaire 15 (2) : 13-20.

MiNISTÈRE DE LA JUSTICE ET MINISTÈRE DU SOLLICITEUR GÉNÉRAL 1986. Politique d'intervention en matière de violence conjugale.

Ministère de la Santé et Des Services sociauX. 1985. Politique d'aide aux femmes violentées.

NiNACS, W. A. 1995. « Empowerment et service social : approches et enjeux », Service social 44 (1) : 69-93.

PARSONS, R. J. 1995. Empowerment based Social Work Practice: a study of Process and Outcomes, texte présenté à la $41^{\mathrm{e}}$ assemblée annuelle du « Council on Social Work Education ». San Diego.

Perkins, D. D. et Zimmerman, M. 1995. «Empowerment Theory, Research and Application », American Journal of Community Psychology 23 (5) : 569579.

PrilleltensKy, I. et GONICK, L. 1996. «Politics change, Oppression remains: on the Psychology and Politics of Oppression », Political Psychology 17 (1) : 127-148.

RAPPAPORT, J. 1985. «The Power of Empowerment Language », Social Policy automne : 15-22.

RAPPAPORT, J. 1987. «Terms of Empowerment/Exemplars of Prevention: Toward a Theory for Community Psychology », American Journal of Community Psychology 15 (2) : 121-145.

Riger, S. 1993. "What's Wrong with Empowerment », American Journal of Community Psychology 21 (3) : 279-292.

RODGERS, K. 1994. «Résultats d'une enquête nationale sur l'agression contre la conjointe ", Juristat, Bulletin de service du Centre canadien de la statistique juridique 14 (9) : 1-22.

Rodwell, C. M. 1996. "An Analysis of the concept of Empowerment", Journal of Advanced Nursing 23 : 305-313.

SHIELDS, L. E. 1995. "Women's Esperiences of the Meaning of Empowerment », Qualitative Health Research 5 (1) : 15-35.

SwilfT, C. et LEVIN, G. 1987. «Empowerment: An emerging mental health technology », Journal of Primary Prevention 8 (1-2) : 71-94.

ZimMERMAN, M. A. 1995. «Psychological Empowerment: Issues and Illustrations », American Journal of Community Psychology 23 (5) : 581-599. 
